\section{Pulmonary Function and Flow- Volume Loop Patterns in Patients With Tracheobronchomalacia: Is There an Independent Effect?}

\section{To the Editor:}

We read with great interest the study by Majid et al describing pulmonary function tests (PFTs) and flow-volume loops in patients with tracheobronchomalacia. ${ }^{1}$ A central question is whether abnormalities in the PFT results and flow-volume loops are attributable to tracheobronchomalacia or concomitant lung disease (eg, COPD or asthma). A secondary question that remains unaddressed is whether such abnormalities in any way predict differential outcomes with attempts to address the tracheobronchomalacia.

In the study by Majid et al, 64 (71\%) of 90 tracheobronchomalacia patients had either COPD or asthma. The PFT results and flow-volume loops from all 90 patients were analyzed in aggregate, irrespective of the presence or absence of additional pulmonary disease. ${ }^{1}$ Majid et al reported that "Among 76 patients with available flowvolume loops, the most frequent finding was low maximum forced expiratory flow, in 62 $(81.6 \%)$ subjects, followed by biphasic morphology in 15 (19.7\%), notched expiratory loop in 7 (9.2\%), and expiratory oscillations in $2(2.6 \%)$. The balance of 13 subjects (17.1\%) had no distinctive flow-volume loop abnormality."1

This study design missed an opportunity to evaluate, through analysis stratified according to comorbid lung pathology, whether tracheobronchomalacia was associated with abnormalities seen on PFT results and flow-volume loops that are independent of the concomitant lung disease. There is currently no clear evidence to support the assumption that tracheobronchomalacia has an independent effect on PFTs and flow-volume loops. Majid et al appropriately point out that central airway collapse from tracheobronchomalacia likely has no effect on expiratory air-flow limitation. ${ }^{1-4}$ Furthermore, several patients with substantial tracheobronchomalacia from MounierKuhn syndrome, but normal lung parenchyma, are reported to have normal PFT results. ${ }^{5}$ Flow-volume loops in COPD and asthma can also be characterized by low maximum forced expiratory flow and biphasic morphology. Could this suggest differential patterns of airways obstruction and in turn convey differential responses to interventions intended to address tracheobronchomalacia? Perhaps the biphasic pattern predicts substantial small-airways obstruction and low probability of improvement with such interventions.

It would be informative if Majid et al could provide data on PFT results and flowvolume loops in patients with only tracheobronchomalacia and no other pulmonary disease, and whether any of the reported PFT results and flow-volume loop findings observed prior to central airway stabilization procedures predict improvement in PFT results, flow-volume loops, or a clinically meaningful outcome.

Michael Eberlein MD PhD

Lung Transplant Program

Division of Pulmonary, Critical Care, and Occupational Medicine

University of Iowa Hospital and Clinics Iowa City, Iowa

Servet Bolukbas MD

Department of Thoracic Surgery Dr Horst Schmidt Klinik

Wiesbaden, Germany

Robert M Reed MD

Division of Pulmonary and Critical Care Medicine

University of Maryland School of Medicine Baltimore Maryland

The authors have disclosed no conflicts of interest.

\section{REFERENCES}

1. Majid A, Sosa AF, Ernst A, Feller-Kopman D, Folch E, Singh A, Gangadharan S. Pulmonary function and flow-volume loop patterns in patients with tracheobronchomalacia. Respir Care 2013;58(9):15211526.

2. Loring SH, O'Donnell CR, Feller-Kopman DJ, Ernst A. Central airway mechanics and flow limitation in acquired tracheobronchomalacia. Chest 2007;131(4):1118-1124.

3. Samad IA, Sanders DE, Suero JT, Woolf CR. The relationship between tracheobronchial collapse and pulmonary function in chronic obstructive pulmonary disease. Dis Chest 1968;53(4):407-412.

4. Litmanovich D, O'Donnell CR, Bankier AA, Ernst A, Millett ME, Loring SH et al. Bronchial collapsibility in healthy volunteers at forced expiration: assessment with multidetector-row CT. Radiology 2010; 257(2):560-567.
5. Ghanei M, Peyman M, Aslani J, Zamel N. Mounier-Kuhn syndrome: a rare cause of severe bronchial dilatation with normal pulmonary function test: a case report. Respir Med 2007;101(8):1836-1839.

Pulmonary Function and FlowVolume Loop Patterns in Patients With Tracheobronchomalacia: Is There an Independent Effect?Reply

In Reply:

We thank Drs Eberlein, Bolukbas, and Reed for their interest in our paper. ${ }^{1}$ We agree that it is often quite difficult to separate the abnormalities seen in pulmonary function test (PFT) results and flow-volume loops that are commonly seen with tracheobronchomalacia from other respiratory conditions that often go hand-in-hand with tracheobronchomalacia. However, we do feel that this represents a real-world scenario, because most patients with symptomatic tracheobronchomalacia will also have a concomitant respiratory disease that also exacerbates their symptoms.

As Eberlein et al correctly point out, the biphasic expiratory curve seems to be more closely related to emphysema than tracheobronchomalacia alone, since $14(87.5 \%)$ of the 16 patients with this spirometric feature had COPD. Only $4(4.4 \%)$ of our patients had no concomitant respiratory diagnosis, again reinforcing that symptomatic tracheobronchomalacia is seldom an isolated disease. In none of these 4 patients were there any characteristic changes in flow-volume loops, except for a low peak flow in 3 patients and a slight coving of the expiratory phase in one patient. The spirometry and lung volumes revealed hyperinflation (high total lung capacity and residual volume) in 2 patients.

Regarding whether our observed patterns may predict a response to treatment, previous studies on central airway stabilization have shown no change in $\mathrm{FEV}_{1}$ despite clinical improvement (as assessed by quality of life, dyspnea scores, and performance scores). This may indicate that the severity of collapse and the presence of symptoms not attributable entirely to another condition are what seem to be the best indicators of response to therapy, regardless of pretreatment PFT results. ${ }^{2,3}$ This last affirmation seems even more plausible when we consider that central airway collapse does 